\title{
Surface correlations for two-dimensional Coulomb fluids in a disc
}

November 21, 2018

\begin{abstract}
B. Jancovici
Laboratoire de Physique Théorique, Bâtiment 210, Université de Paris-Sud, 91405 Orsay, France (Unité Mixte de Recherche no.8627-CNRS); e-mail: Bernard.Jancovici @th.u-psud.fr
\end{abstract}

\begin{abstract}
After a brief review of previous work, two exactly solvable two-dimensional models of a finite Coulomb fluid in a disc are studied. The charge correlation function near the boundary circle is computed. When the disc radius is large compared to the bulk correlation length, a correlation function of the surface charge density can be defined. It is checked, on the solvable models, that this correlation function does have the generic long-range behaviour, decaying as the inverse square distance, predicted by macroscopic electrostatics. In the case of a two-component plasma (Coulomb fluid made of two species of particles of opposite charges), the density correlation function on the boundary circle itself is conjectured to have a temperature-independent behaviour, decaying as the -4 power of the distance.
\end{abstract}

LPT Orsay 02-20 


\section{Introduction}

This paper is dedicated to Jean-Pierre Hansen on the occasion of his 60th birthday. Although Jean-Pierre and I have coauthored only one paper [1], some 30 years ago, we share a longstanding interest for Coulomb fluids. Here is a contribution to this thriving domain of research. It consists of a far from exhaustive minireview of previous work on exactly solvable two-dimensional models of Coulomb fluids, followed by an original part about a finite Coulomb fluid in a disc.

\subsection{A brief review}

The classical (i.e. non-quantum) statistical mechanics of some two-dimensional models of Coulomb fluids is exactly solvable. These models have an intrisic interest: this is the only case of solvable models for a continuous (i.e. not on a lattice) fluid, in more than one dimension. Furthermore, these models can be used as a testbench for a variety of generic properties of Coulomb fluids. For mimicking three-dimensional Coulomb fluids in two dimensions, one must use the two-dimensional Coulomb interaction which is logarithmic: the interaction energy between two unit charges at a distance $r$ of each other is chosen as $\ln (L / r)$, where $L$ is an arbitrary length which only fixes the zero of energy. Indeed, this interaction is the two-dimensional solution of the Poisson equation

$$
\Delta \ln \frac{L}{r}=-2 \pi \delta(\mathbf{r})
$$

Of course, these logarithmic models do not describe "real" charged particles, such as electrons, confined in a plane, which nevertheless interact through the three-dimensional Coulomb potential $1 / r$.

Two models are of special interest: The one-component plasma (OCP), or jellium, is made of one species of particles of charge $q$, embedded in a uniformly charged background of the opposite sign. The two-component plasma (TCP), or Coulomb gas, is made of two species of particles, with opposite charges $\pm q$. At the inverse temperature $\beta$, the dimensionless coupling constant can be chosen as $\Gamma=\beta q^{2}$. It has the remarkable property of being independent of the density, and this results into a simple equation of state [2, 3] for both models

$$
\beta p=\left(1-\frac{\Gamma}{4}\right) n
$$

where $p$ is the pressure and $n$ is the density.

In the case of the TCP, the model (with pure Coulomb interactions) becomes unstable againt the collapse of positive-negative pairs for $\Gamma \geq 2$, and the equation of state (1.2) is valid only for $\Gamma \leq 2$. If some short-range repulsion (for instance hard cores of diameter

a) is introduced, the collapse at $\Gamma=2$ is suppressed and the temperature can be further lowered. For small values of the dimensionless density $n a^{2}$, the famous Kosterlitz-Thouless phase transition [46] of infinite order occurs, between a conducting high-temperature phase and a dielectric low-temperature phase, at a density-dependent temperature with a corresponding $\Gamma$ close to 4 .

Finite-size two-dimensional Coulomb fluids exhibit universal properties [0] related to conformal invariance. This will not be reviewed here. 
For the special value $\Gamma=2$, the OCP [8,9] and TCP [10] are fully exactly solvable: The thermodynamic functions and the correlation functions are obtainable.

For the OCP, it is convenient to define the Ursell function $U$ as

$$
U\left(\mathbf{r}, \mathbf{r}^{\prime}\right)=n^{(2)}\left(\mathbf{r}, \mathbf{r}^{\prime}\right)-n^{2}
$$

where $n$ is the one-body density and $n^{(2)}\left(\mathbf{r}, \mathbf{r}^{\prime}\right)$ is the two-body density. At $\Gamma=2$, in the canonical ensemble, the OCP maps on a system of free fermions in a magnetic field and one finds

$$
U\left(\mathbf{r}, \mathbf{r}^{\prime}\right)=-n^{2} \exp \left(-\pi n\left|\mathbf{r}-\mathbf{r}^{\prime}\right|^{2}\right)
$$

This correlation function has a fast Gaussian decay on a lengthscale of the order of $(\pi n)^{-1 / 2}$. Several generic sum rules can be checked. The zeroth and second moments of $U$ obey the two Stillinger-Lovett sum rules [11] which express perfect screening of a charged particle of the fluid and perfect screening of an added infinitesimal test charge, respectively. The fourth moment obeys the general expression derived, in terms of the compressibility, by Vieillefosse and Hansen [12],

$$
n\left(\frac{\pi \Gamma}{4}\right)^{2} \int U\left(\mathbf{r}, \mathbf{r}^{\prime}\right)\left|\mathbf{r}-\mathbf{r}^{\prime}\right|^{4} \mathrm{~d} \mathbf{r}=-\beta\left(\frac{\partial p}{\partial n}\right)_{T}
$$

and the sixth moment obeys a recently derived expression 13.

$$
n^{2}\left(\frac{\pi \Gamma}{2}\right)^{3} \int U\left(\mathbf{r}, \mathbf{r}^{\prime}\right)\left|\mathbf{r}-\mathbf{r}^{\prime}\right|^{6} \mathrm{~d} \mathbf{r}=\frac{3}{4}(\Gamma-6)(8-3 \Gamma) .
$$

For the TCP, the Ursell functions $U$ depend on the species of the two particles which are involved. They must be defined as

$$
U_{s s^{\prime}}\left(\mathbf{r}, \mathbf{r}^{\prime}\right)=n_{s s^{\prime}}^{(2)}\left(\mathbf{r}, \mathbf{r}^{\prime}\right)-n_{s} n_{s^{\prime}}
$$

where $s, s^{\prime}= \pm 1$ denote the signs of the particles, $n_{+}=n_{-}=n / 2$ are the one-body densities of each species, $n$ the total one-body density, and $n_{s s^{\prime}}^{(2)}\left(\mathbf{r}, \mathbf{r}^{\prime}\right)$ is the two-body density for particles of species $s$ and $s^{\prime}$, respectively. At $\Gamma=2$, in the grand canonical ensemble, the TCP maps on a system of free fermions. A control parameter is a properly rescaled fugacity $m$, which has the dimension of an inverse length. Although the density diverges at $\Gamma=2$, the Ursell functions remain finite. They are

$$
\begin{aligned}
& U_{s s}\left(\mathbf{r}, \mathbf{r}^{\prime}\right)=-\left(\frac{m^{2}}{2 \pi}\right)^{2}\left[K_{0}\left(m\left|\mathbf{r}-\mathbf{r}^{\prime}\right|\right)\right]^{2} \\
& U_{s-s}\left(\mathbf{r}, \mathbf{r}^{\prime}\right)=\left(\frac{m^{2}}{2 \pi}\right)^{2}\left[K_{1}\left(m\left|\mathbf{r}-\mathbf{r}^{\prime}\right|\right)\right]^{2}
\end{aligned}
$$

where $K_{0}$ and $K_{1}$ are modified Bessel functions, which have an exponential decay; the correlation length is $1 /(2 m)$. The short- distance behaviours of these Ursell functions confirm general predictions of Hansen and Viot [14]. The pair distribution function for particles of opposite signs, dominated at short distances by the Bolzmann factor of the 
Coulomb potential, should behave like $\left|\mathbf{r}-\mathbf{r}^{\prime}\right|^{-\Gamma}$; the $\left|\mathbf{r}-\mathbf{r}^{\prime}\right|^{-2}$ behaviour of $U_{s-s}$ fits with this form. However, although the pair distribution function for particles of the same sign should behave like $\left|\mathbf{r}-\mathbf{r}^{\prime}\right|^{\Gamma}$ for $\Gamma<1$, this repulsive behaviour has been predicted to be weakened into $\left|\mathbf{r}-\mathbf{r}^{\prime}\right|^{2-\Gamma}$ for $1<\Gamma<2$. because of screening by a third particle; the logarithmic behaviour of the Bessel function $K_{0}$ in $U_{s s}$ can be considered as a limiting case when $\Gamma=2$.

The Ursell functions for the TCP can be combined into a charge correlation function

$$
U_{\rho}=2 q^{2}\left(U_{++}-U_{-+}\right)
$$

and a density correlation function

$$
U_{n}=2\left(U_{++}+U_{-+}\right) .
$$

Several generic sum rules can be checked on these correlation functions. The zeroth and second moment of the charge correlation function (1.9) obey the Stillinger-Lovett sum rules. The zeroth moment of the density correlation function (1.10) obeys the usual compressibility sum rule, while its second moment obeys a recently dicovered sum rule 15, 16] which seems to be specific to the two-dimensional point-particle TCP:

$$
\int U_{n}\left(\mathbf{r}, \mathbf{r}^{\prime}\right)\left|\mathbf{r}-\mathbf{r}^{\prime}\right|^{2} \mathrm{~d} \mathbf{r}=\frac{1}{12 \pi\left[1-(\Gamma / 4)^{2}\right]}
$$

Up to two years ago,there were exact results only at $\Gamma=2$. Then a major breakthrough occurred: Šamaj et al. [17] succeeded in deriving the thermodynamic properties of the TCP in the whole range of stability of the model, $\Gamma<2$. This was achieved by using a mapping on the sine-Gordon field theory and results known for the latter. The surface tension along a wall could also be obtained in the cases of an ideal conductor wall [18] and of an ideal dielectric wall [19]. However, although results have been obtained for the asymptotic behaviour of the correlation functions [7, 20], there are no simple expressions for these correlation functions. In the following, we shall consider only the simple case $\Gamma=2$, and the weak-coupling (high-temperature) limit $\Gamma \rightarrow 0$.

At $\Gamma=2$, many exact results are available for the OCP and the TCP in a variety of geometries with walls or on a curved surface. These results will not be described in detail, the interested reader might look on cond-mat for Forrester, Jancovici, Téllez .... Here, we only review the case of a Coulomb fluid living in a half-plane bounded by a rectilinear hard wall. This hard wall can be taken as the $y$ axis, and the fluid is supposed to occupy the half-plane $x \geq 0$. The position $\mathbf{r}$ of a particle is defined by its Cartesian coordinates $x$ and $y$. Now, in the definitions of the Ursell functions, it must be understood that the one-body densities are functions of the distance to the wall, i.e. in (1.3) $n^{2}$ must be replaced by $n(x) n\left(x^{\prime}\right)$, and in (1.7) $n_{s} n_{s^{\prime}}$ must be replaced by $n_{s}(x) n_{s^{\prime}}\left(x^{\prime}\right)$. The Ursell functions depend on $x, x^{\prime}$, and $\left|y-y^{\prime}\right|$.

For this half-plane geometry, a generic behaviour of the charge correlations near the wall 21 23 results from the assumption that the fluid is a conductor obeying the laws of macroscopic electrostatics. The charge correlation function along the wall now is longranged, with only an algebraic asymptotic decay

$$
U_{\rho}\left(x, x^{\prime},\left|y-y^{\prime}\right|\right) \sim \frac{f\left(x, x^{\prime}\right)}{\left(y-y^{\prime}\right)^{2}}
$$


when $\left|y-y^{\prime}\right|$ is large compared to the microscopic scale (the bulk correlation length). $f\left(x, x^{\prime}\right)$ is a function which is localized near the wall (it has a fast decay as $x$ or $x^{\prime}$ increase beyond the microscopic scale), and $f$ obeys the sum rule

$$
\beta \int_{0}^{\infty} \mathrm{d} x \int_{0}^{\infty} \mathrm{d} x^{\prime} f\left(x, x^{\prime}\right)=-\frac{1}{2 \pi^{2}} .
$$

(11.12) and (11.13) can be reexpressed by writing that there is a surface charge density $\sigma(y)$ with a correlation function obeying

$$
\beta<\sigma(y) \sigma\left(y^{\prime}\right)>=-\frac{1}{2 \pi^{2}\left(y-y^{\prime}\right)^{2}}
$$

At $\Gamma=2$ and in the limit $\Gamma \rightarrow 0$, along a hard wall, the charge correlation functions $q^{2} U$ of the OCP [21] and $U_{\rho}$ of the TCP [7] do have an asymptotic behaviour in agreement with (1.14).

For this half-plane geometry, it has been observed [7] that the density correlation function of the TCP near the wall is also long-ranged and that $U_{n}\left(x=0, x^{\prime}=0,\left|y-y^{\prime}\right|\right)$ has the same asymptotic behaviour at $\Gamma=2$ and as $\Gamma \rightarrow 0$ :

$$
U_{n}\left(x=0, x^{\prime}=0,\left|y-y^{\prime}\right|\right) \sim \frac{1}{2 \pi^{2}\left(y-y^{\prime}\right)^{4}} .
$$

It is tempting to conjecture that (1.15) is valid at any temperature.

\subsection{The disc geometry}

We now come to the original part of the present paper. In the above, it was always found that the laws of macroscopic electrostatics about charge correlations were satisfied by the exactly solvable two-dimensional models, when the lengths under consideration are large compared to the microscopic scale. Recently, however, a counterexample was found [24]. A short-circuited circular capacitor was considered: A two-dimensional Coulomb fluid fills a disk of radius $R_{1}$ and the infinite region outside a concentric circle of larger radius $R_{2}$, while the annulus between $R_{1}$ and $R_{2}$ is empty, and the two filled regions are allowed to freely exchange charged particles. This geometry is exactly solvable at $\Gamma=2$ for both the OCP and the TCP. The charge $Q$ on the inner disk fluctuates. Even for macroscopic values of $R_{1}, R_{2}$, with $R_{2} / R_{1}>1$, it was found that the variance $\left\langle Q^{2}>-<Q\right\rangle^{2}$ differs from the value predicted by using linear response theory and macroscopic electrostatics

$$
\beta\left(<Q^{2}>-<Q>^{2}\right)=\frac{1}{\ln \frac{R_{2}}{R_{1}}} .
$$

This, at first sight surprising, disagreement can be explained, on second thought, when it is noted that the fluctuations (1.16) involve only a small number of particles, because, at $\Gamma=2,\left(<Q^{2}>-<Q>^{2}\right) / q^{2}$ is of order unity. Although, for one disc alone, i.e. in the limit $R_{2} \rightarrow \infty$, no charge fluctuations are found for the solvable models (except in one very special case), in agreement with the limit $R_{2} \rightarrow \infty$ in (1.16), the failure of (1.16) for $R_{2} / R_{1}$ finite casts a reasonable doubt on the predictions of macroscopic electrostatics in 
the disc geometry. The subject of the present paper is to investigate the surface charge correlations of the OCP and TCP in a disc, at $\Gamma=2$. It will be shown that macroscopic electrostatics does hold for the present problem.

Thus, we consider a Coulomb fluid in a disc of radius $R$, bounded by a hard wall. It is convenient to put the origin at the centre of the disc, and to use polar coordinates $(r, \varphi)$. The disc may be either insulated, with for simplicity a vanishing total charge, or grounded. In both cases, from linear response theory and macroscopic electrostatics [23] one finds that the correlation function of the surface charge density $\sigma(\varphi)$ is given by

$$
\beta<\sigma(\varphi) \sigma\left(\varphi^{\prime}\right)>=-\frac{1}{2 \pi^{2}[2 R \sin (\theta / 2)]^{2}}
$$

where $\theta=\varphi-\varphi^{\prime}$ is the angular distance between the two points. Actually, the correlation function in (1.17) is defined from the microscopic charge Ursell function $U_{\rho}\left(r, r^{\prime},|\theta|\right)$, which, for $R$ and $R|\theta|$ large compared to the microscopic scale, is localized near the boundary circle (it has a fast decay as $x=R-r$ or $x^{\prime}=R-r^{\prime}$ increase beyond the microscopic scale). The relation is

$$
<\sigma(\varphi) \sigma\left(\varphi^{\prime}\right)>=\int_{0}^{\infty} \mathrm{d} x \int_{0}^{\infty} \mathrm{d} x^{\prime} U_{\rho}\left(r, r^{\prime},|\theta|\right) .
$$

Also, possible oscillations as a function of $\theta$ on a microscopic scale are washed out in the definition of the surface charge density. (1.17) is a generalization of the rectilinear wall case (1.14), which is retrieved from (1.17) in the limit $R \rightarrow \infty, \theta \rightarrow 0$, at a fixed value of $R \theta$ which becomes $y-y^{\prime}$.

In section 2 , it will be shown that (1.17) is obeyed in the case of the OCP at $\Gamma=2$. In section 3, it will be shown that $(1.17)$ is obeyed in the case of the TCP at $\Gamma=2$. A generalization to the disc geometry of the density correlation function on the wall for the TCP (1.15) will also be presented. The high-temperature limit $\Gamma \rightarrow 0$ will be briefly reviewed in section 4 .

\section{One-component plasma at $\Gamma=2$ in a disc}

The canonical ensemble is used. There are $N$ particles of charge $q$ in a disc of radius $R$. Thus, the average density is $n=N /\left(\pi R^{2}\right)$. A uniformly charged background insures that the total charge vanishes. It is convenient to choose the unit of length as $R / \sqrt{N}$. In these units, $\pi n=1$. The general formalism [21] for the OCP in a disk at $\Gamma=2$ expresses the density Ursell function $U\left(r, r^{\prime},|\theta|\right)=n^{(2)}\left(r, r^{\prime},|\theta|\right)-n(r) n\left(r^{\prime}\right)$ in terms of an auxiliary function

$$
K(w)=\sum_{l=0}^{N-1} \frac{w^{l}}{\gamma(l+1, N)}
$$

where $w=r r^{\prime} \exp (\mathrm{i} \theta)$ and $\gamma(l+1, N)$ is an incomplete gamma function [25], as

$$
U\left(r, r^{\prime},|\theta|\right)=-n^{2} \exp \left(-r^{2}-r^{\prime 2}\right)|K(w)|^{2}
$$

We are interested in the behaviour of (2.2) when $r$ and $r^{\prime}$ are close to $R$ and $R \theta$ is much larger than the microscopic scale $n^{-1 / 2}$, i.e. for $N$ large and a non-zero value of $\theta$. 
A related problem has been previously solved by Choquard et al. [26]. They considered the simpler case when the confining circular background extends well beyond the circular blob formed by the particles. Then, in (2.1), the incomplete gamma function is replaced by the complete one $\Gamma(l+1)=l$ ! and the sum itself can be expressed in terms of another incomplete gamma function, from which the surface behaviour of $U$ could be obtained (the surface behaviour of $U$ has also been obtained [27] in the case of an elliptical blob of particles). In our present case, since for $r$ and $r^{\prime}$ close to $R$ the sum in (2.1) can be seen to be dominated by values of $l$ close to $N$, we use the asymptotic expression [25] $\gamma(l+1, l) \sim(1 / 2) l$ !. Thus our $K$ is just twice the one of Choquard et al. Although we have not been able to make a rigorous mathematical proof, the validity of this procedure has been checked on Mathematica, in the cases $r=r^{\prime}=R, \theta=\pi / 2$ or $\theta=\pi$, by numerical evaluations of a sum equivalent to (2.1).

Following Choquard et al., we can now express our $K$ as [25]

$$
K(w)=2 \sum_{l=0}^{N-1} \frac{w^{l}}{l !}=\frac{2}{(N-1) !} \exp (w) \Gamma(N, w)
$$

where $\Gamma(N, w)$ is the incomplete gamma function

$$
\Gamma(N, w)=\int_{w}^{\infty} \mathrm{d} t \mathrm{e}^{-t} t^{N-1}
$$

The asymptotic form of (2.4), in the present case of $|w-N| \gg \sqrt{N}$ and $N \rightarrow \infty$, can be obtained by rewriting (2.4) as

$$
\Gamma(N, w)=\int_{w}^{\infty} \mathrm{d} t \frac{t}{N-1-t} \frac{\mathrm{d}}{\mathrm{d} t}\left(t^{N-1} \mathrm{e}^{-t}\right)
$$

and integrating by parts, with the result

$$
\frac{\exp (w)}{(N-1) !} \Gamma(N, w)=\frac{w^{N}}{(N-1) !(w-N+1)}\left[1+O\left(\frac{1}{N}\right)\right]
$$

in agreement with an asymptotic expansion by Tricomi (see 25]). In the case of two particles on the circle of radius $R$, since in our units $R^{2}=N, w=N \exp (\mathrm{i} \theta)$, and from (2.3) and (2.6) one finds

$$
\mathrm{e}^{-N} K\left(N \mathrm{e}^{\mathrm{i} \theta}\right) \sim \frac{2 \mathrm{e}^{-N} N^{N}}{N !} \frac{\mathrm{e}^{\mathrm{i} N \theta}}{\mathrm{e}^{\mathrm{i} \theta}-1}
$$

Using Stirling's formula for $N$ ! in (2.7) gives

$$
\mathrm{e}^{-N} K\left(N \mathrm{e}^{\mathrm{i} \theta}\right) \sim\left(\frac{2}{\pi N}\right)^{1 / 2} \frac{\mathrm{e}^{\mathrm{i} N \theta}}{\mathrm{e}^{\mathrm{i} \theta}-1} .
$$

Using (2.8) in (2.2) and reestablishing an arbitrary unit of length such that $\pi n$ is no longer 1 , gives the correlation function on the circle

$$
U(R, R, \theta) \sim-n \frac{2}{\pi^{2}} \frac{1}{\left(2 R \sin \frac{\theta}{2}\right)^{2}} .
$$


In the case when the two particles are at small distances $x$ and $x^{\prime}$ from the boundary, minor modifications after (2.6) give, in the large- $N$ limit

$$
U\left(r, r^{\prime}, \theta\right) \sim-n \frac{2}{\pi^{2}} \frac{\exp \left(-2 \pi n x^{2}-2 \pi n x^{\prime 2}\right)}{\left(2 R \sin \frac{\theta}{2}\right)^{2}} .
$$

Integrating $U_{\rho}=q^{2} U$ with respect to $x$ and $x^{\prime}$ does give a correlation function of the surface charge density of the form (1.17) (here $\beta q^{2}=2$ ), in agreement with macroscopic electrostatics.

The case of a rectilinear hard wall [21] is retrieved from (2.10) by taking the limit $R \rightarrow \infty, \theta \rightarrow 0$, at a fixed value of $R \theta$ which becomes $y-y^{\prime}$. Then, in agreement with (1.12),

$$
U_{\rho}\left(x, x^{\prime},\left|y-y^{\prime}\right|\right) \sim-q^{2} n \frac{\exp \left(-2 \pi n x^{2}-2 \pi n x^{\prime 2}\right)}{\left(y-y^{\prime}\right)^{2}}
$$

and (1.14) is satisfied.

In the derivation of the present results, we have replaced $\gamma(l+1, N)$ by $(1 / 2) l$ ! without a rigorous mathematical justification. However, the validity of (2.9) and (2.10) is supported by several checks. First, the correct result (2.11) was retrieved in the limiting case of a rectilinear wall. Second, using the exact expression (2.1), Choquard et al. 28] have shown without approximations that, in the large- $N$ limit,

$$
-\frac{1}{2 \pi R^{2}} \int \mathrm{d} \mathbf{r} \mathrm{d} \mathbf{r}^{\prime}\left|\mathbf{r}-\mathbf{r}^{\prime}\right|^{2} U\left(\mathbf{r}, \mathbf{r}^{\prime}\right)=\frac{1}{\pi}
$$

and that the surface contribution to $(2.12)$ is $1 /(2 \pi)$; this is in agreement with what is obtained by using for $U$ near the surface the expression (2.10).

\section{Two-component plasma at $\Gamma=2$ in a disc}

The particles are confined by a hard wall in a disc of radius $R$. A grand canonical ensemble restricted to neutral configurations is used. Both species of particles, of respective charges $q$ and $-q$, have the same rescaled fugacity $m$. The general formalism [10] expresses the Ursell functions $U_{s s^{\prime}}\left(r, r^{\prime},|\theta|\right)$ in terms of Green functions $G_{s s^{\prime}}\left(\mathbf{r}, \mathbf{r}^{\prime}\right)$ as

$$
U_{s s^{\prime}}\left(r, r^{\prime},|\theta|\right)=-s s^{\prime} m^{2}\left|G_{s s^{\prime}}\left(\mathbf{r}, \mathbf{r}^{\prime}\right)\right|^{2} .
$$

Because of the symmetry between positive and negative particles, we only need $G_{++}$and $G_{-+}$which, for $r, r^{\prime}<R$, are determined by

$$
\left(m^{2}-\Delta\right) G_{++}\left(\mathbf{r}, \mathbf{r}^{\prime}\right)=m \delta\left(\mathbf{r}-\mathbf{r}^{\prime}\right)
$$

and

$$
G_{-+}\left(\mathbf{r}, \mathbf{r}^{\prime}\right)=-\frac{\exp (\mathrm{i} \varphi)}{m}\left(\frac{\partial}{\partial r}+\frac{\mathrm{i}}{r} \frac{\partial}{\partial \varphi}\right) G_{++}\left(\mathbf{r}, \mathbf{r}^{\prime}\right)
$$


In infinite space the solution of (3.2) is $[m /(2 \pi)] K_{0}\left(m\left|\mathbf{r}-\mathbf{r}^{\prime}\right|\right)$. In a disc, it is appropriate to use polar coordinates and to write the solution as an expansion of the form

$$
G_{++}\left(r, \varphi ; r^{\prime}, \varphi^{\prime}\right)=\frac{m}{2 \pi} \sum_{l=-\infty}^{\infty}\left[I_{l}\left(m r^{\prime}\right) K_{l}(m r)+a_{l} I_{l}\left(m r^{\prime}\right) I_{l}(m r)\right] \exp \left[\mathrm{i} l\left(\varphi-\varphi^{\prime}\right)\right] \quad\left(r^{\prime}<r<R\right)
$$

where the first term in the sum is the expansion of $[m /(2 \pi)] K_{0}\left(m\left|\mathbf{r}-\mathbf{r}^{\prime}\right|\right)$ and the second term is a "reflected" contribution due to the wall; $I_{l}$ and $K_{l}$ are modified Bessel functions and $a_{l}$ is a coefficient to be determined by the boundary conditions. (3.3) gives

$$
\begin{aligned}
& G_{-+}\left(r, \varphi ; r^{\prime}, \varphi^{\prime}\right)=\frac{m}{2 \pi} \sum_{l=-\infty}^{\infty}\left[I_{l}\left(m r^{\prime}\right) K_{l+1}(m r)\right. \\
& \left.-a_{l} I_{l}\left(m r^{\prime}\right) I_{l+1}(m r)\right] \exp \left[\mathrm{i}(l+1) \varphi-\mathrm{i} l \varphi^{\prime}\right] \quad\left(r^{\prime}<r<R\right)
\end{aligned}
$$

For $r^{\prime}<R, r>R, m$ must be replaced by 0 in (3.2), and, as functions of $\mathbf{r}, G_{++}$depends only on $z=r \exp (\mathrm{i} \varphi), G_{-+}$depends only on $\bar{z}=r \exp (-\mathrm{i} \varphi)$, and they must vanish at infinity. At $r=R, G_{++}$and $G_{-+}$must be continuous. These conditions impose that the $l \geq 0$ terms in $G_{++}\left(R, \varphi ; r^{\prime}, \varphi^{\prime}\right)$ and the terms $l<0$ in $G_{-+}\left(R, \varphi ; r^{\prime}, \varphi^{\prime}\right)$ vanish. Therefore, the coefficient $a_{l}$ in (3.4) and (3.5) is

$$
a_{l}=-\frac{K_{l}(m R)}{I_{l}(m R)} \text { if } l \geq 0, \quad a_{l}=\frac{K_{l+1}(m R)}{I_{l+1}(m R)} \text { if } l<0 .
$$

We are interested in the behaviours of (3.4) and (3.5) when the disc is much larger that the bulk correlation length $(m R \gg 1), \theta=\varphi-\varphi^{\prime}$ has a fixed (non-zero) value, and $r$ and $r^{\prime}$ are close to $R$.

Let us start with the case when the two points are on the disc. In this limiting case, when the Wronskian relation [25] $I_{l}(m R) K_{l+1}(m R)+I_{l+1}(m R) K_{l}(m R)=1 /(m R)$ is taken into account, and after $l$ has been changed into $-l$, (3.4) and (3.6) give

$$
G_{++}\left(R, \varphi ; R, \varphi^{\prime}\right)=\frac{1}{2 \pi R} \sum_{l=1}^{\infty} \frac{I_{l}(m R)}{I_{l-1}(m R)} \exp \left[-\mathrm{i} l\left(\varphi-\varphi^{\prime}\right)\right]
$$

while, when that same Wronskian relation is used, (3.5) and (3.6) give

$$
\left.G_{-+}\left(R, \varphi ; R, \varphi^{\prime}\right)=\frac{1}{2 \pi R} \sum_{l=0}^{\infty} \exp \left[\mathrm{i}(1+l) \varphi-\mathrm{i} l \varphi^{\prime}\right)\right]
$$

In the large- $m R$ limit, using in (3.7) the large-argument asymptotic expansions of the Bessel functions [29] give, up to order $1 / R^{2}$,

$$
\frac{I_{l}(m R)}{I_{l-1}(m R)}=1+\frac{1-2 l}{2 m R}+\frac{3-8 l+4 l^{2}}{8(m R)^{2}}+\ldots .
$$

Although the sums on $l$ which appear in (3.8), and in (3.7) when (3.9) is used, are not convergent, they can be given a meaning in the sense of distributions; the obvious recipe 
is to insert a convergence factor $p^{l}$, with $|p|<1$, in each term, and to take the limit $p \rightarrow 1$ after the summation. One finds, up to order $1 / R^{3}$,

$$
\begin{aligned}
G_{++}\left(R, \varphi ; R, \varphi^{\prime}\right) & =\frac{1}{2 \pi R} \frac{\mathrm{e}^{-\mathrm{i} \theta}}{1-\mathrm{e}^{-\mathrm{i} \theta}}\left[1-\frac{1}{2 m R} \frac{1+\mathrm{e}^{-\mathrm{i} \theta}}{1-\mathrm{e}^{-\mathrm{i} \theta}}\right. \\
& \left.+\frac{1}{8(m R)^{2}} \frac{-1+6 \mathrm{e}^{-\mathrm{i} \theta}+3 \mathrm{e}^{-2 \mathrm{i} \theta}}{\left(1-\mathrm{e}^{-\mathrm{i} \theta}\right)^{2}}+\ldots\right]
\end{aligned}
$$

where $\theta=\varphi-\varphi^{\prime}$, and

$$
G_{-+}\left(R, \varphi ; R, \varphi^{\prime}\right)=\frac{\mathrm{e}^{\mathrm{i} \varphi}}{2 \pi R} \frac{1}{1-\mathrm{e}^{\mathrm{i} \theta}} .
$$

It might be noted that the expression (3.11) is exact, without any large- $m R$ expansion. Finally, using (3.10) or (3.11) in (3.1) gives

$$
U_{++}(R, R,|\theta|)=-\frac{m^{2}}{4 \pi^{2}\left(2 R \sin \frac{\theta}{2}\right)^{2}}+\frac{1}{4 \pi^{2}\left(2 R \sin \frac{\theta}{2}\right)^{4}}+\ldots
$$

up to order $1 / R^{4}$, and

$$
U_{-+}(R, R,|\theta|)=\frac{m^{2}}{4 \pi^{2}\left(2 R \sin \frac{\theta}{2}\right)^{2}} .
$$

More generally, if the two points are at small distances $x$ and $x^{\prime}$ from the boundary, using the large-argument asymptotic expansions of the Bessel functions in (3.4) and (3.5) (where the first term in the sum, which comes from the expansion of $K_{0}$, gives a shortranged contribution which can be omitted) gives, when only the leading term is kept,

$$
G_{++}\left(R-x, \varphi ; R-x^{\prime}, \varphi^{\prime}\right) \sim \frac{\mathrm{e}^{-m\left(x+x^{\prime}\right)}}{2 \pi R} \frac{\mathrm{e}^{-\mathrm{i} \theta}}{1-\mathrm{e}^{-\mathrm{i} \theta}}
$$

and

$$
G_{-+}\left(R-x, \varphi ; R-x^{\prime}, \varphi^{\prime}\right) \sim \frac{\mathrm{e}^{-m\left(x+x^{\prime}\right)+\mathrm{i} \varphi}}{2 \pi R} \frac{1}{1-\mathrm{e}^{\mathrm{i} \theta}}
$$

The Ursell functions become

$$
U_{++}\left(R-x, R-x^{\prime},|\theta|\right) \sim-\frac{m^{2} \mathrm{e}^{-2 m\left(x+x^{\prime}\right)}}{4 \pi^{2}\left(2 R \sin \frac{\theta}{2}\right)^{2}}
$$

and

$$
U_{-+}\left(R-x, R-x^{\prime},|\theta|\right) \sim \frac{m^{2} \mathrm{e}^{-2 m\left(x+x^{\prime}\right)}}{4 \pi^{2}\left(2 R \sin \frac{\theta}{2}\right)^{2}} .
$$

The charge correlation function is

$$
U_{\rho}\left(R-x, R-x^{\prime},|\theta|\right)=2 q^{2}\left[U_{++}\left(R-x, R-x^{\prime},|\theta|\right)-U_{-+}\left(R-x, R-x^{\prime},|\theta|\right)\right] .
$$

Using (3.16) and (3.17) in (3.18) and integrating it on $x$ and $x^{\prime}$ does give a correlation function of the surface charge density of the form (1.17) (here $\beta q^{2}=2$ ), in agreement with macroscopic electrostatics. 
The density correlation function on the wall is

$$
U_{n}(R, R,|\theta|)=2\left[U_{++}(R, R,|\theta|)+U_{-+}(R, R,|\theta|)\right] .
$$

In (3.19), the leading contribution to $U_{++}$is cancelled by $U_{-+}$and it is necessary to use the full expansion (3.12), together with (3.13) for obtaining the leading term of $U_{n}$ as

$$
U_{n}(R, R,|\theta|) \sim \frac{1}{2 \pi^{2}\left(2 R \sin \frac{\theta}{2}\right)^{4}} .
$$

(3.20) is the generalization to the case of a circular wall of the rectilinear wall result (1.15).

A mathematical justification of some of the heuristic steps used in the above derivations is given in the Appendix.

\section{High-temperature limit $\Gamma \rightarrow 0$ in a disc}

For both the OCP and the TCP, the high-temperature limit $\Gamma \rightarrow 0$ is described by the Debye-Hückel theory. The case of a disc has already been investigated by Choquard et al. [30. They have shown that, when $x=R-r$ and $x^{\prime}=R-r^{\prime}$ are small, the charge correlation function ist

$$
U_{\rho}\left(r, r^{\prime},|\theta|\right) \sim-\frac{q^{2} n \mathrm{e}^{-\kappa\left(x+x^{\prime}\right)}}{\pi\left(2 R \sin \frac{\theta}{2}\right)^{2}}
$$

where $\kappa=\left(2 \pi \beta q^{2} n\right)^{1 / 2}$ is the the inverse Debye length. Integrating (4.1) on $x$ and $x^{\prime}$ does give a correlation function of the surface charge density of the form (11.17), in agreement with macroscopic electrostatics.

In the case of the TCP, the density correlation function $U_{n}$ is also of interest. Since the Debye-Hückel theory gives a vanishing result (the contributions from the Debye-Hückel $U_{s s}$ and $U_{s-s}$ cancel each other), it is necessary to go beyond the Debye-Hückel theory and to take into account the next term in the renormalized Mayer expansion [16, 20], which is proportional to the square of the Debye-Hückel $U_{\rho}$. One finds

$$
U_{n}\left(R-x, R-x^{\prime},|\theta|\right) \sim \frac{\mathrm{e}^{-2 \kappa\left(x+x^{\prime}\right)}}{2 \pi^{2}\left(2 R \sin \frac{\theta}{2}\right)^{4}} .
$$

On the wall itself, (4.2) is the same as in the case $(3.20)$ of $\Gamma=2$.

\section{Conclusion}

Since, in two dimensions, we could have some doubts about the validity of macroscopic electrostatics, which is used for deriving the correlation function (1.17) of the surface

\footnotetext{
${ }^{1}$ Actually, Choquard et al. have given, instead of (4.1), a more general expression involving the arbitrary length $L$ in the logarithmic potential $\ln (L / r)$. We have argued [24] that, in two dimensions, there should be no fluctuations of the total charge on the disc, even in a grand canonical ensemble, and that this condition imposes that the limit $L \rightarrow \infty$ should be taken. Then, the expression of Choquard et al. reduces to $(4.1)$.
} 
charge density for a Coulomb fluid in a disc, we have checked this correlation function on exactly solvable models, the OCP and TCP at $\Gamma=2$. That correlation function had also been obtained in the Debye-Hückel theory [30], i.e. in the weak-coupling $\Gamma \rightarrow 0$ limit. Thus, these calculations indicate that macroscopic electrostatics is valid for describing the correlation function of the surface charge density. Incidentally, this correlation function (times $\beta$ ) can be formally written as a Fourier series

$$
\beta<\sigma(\varphi) \sigma\left(\varphi^{\prime}\right)>=-\frac{1}{2 \pi^{2}[2 R \sin (\theta / 2)]^{2}}=\frac{1}{2 \pi^{2} R^{2}} \sum_{l=1}^{\infty} l \cos (l \theta) .
$$

This Fourier series has no $l=0$ term, another indication that the total charge has no fluctuations.

In the case of a TCP, the density correlation function on the wall has the same form (3.20) at $\Gamma=2$ and in the high-temperature limit $\Gamma \rightarrow 0$. It is tempting to conjecture that this form is valid at any temperature, at least in the range $\Gamma \leq 2$. A similar universality of (1.15) had been conjectured [7] in the case of a rectilinear wall. Proving (or disproving) these conjectures is an open problem.

\section{Appendix}

In section 3, large-argument expansions of Bessel functions have been used, although these expansions have been inserted into infinite series involving also large values of the index $l$. Furthermore, these series in $l$ have been made to converge only through the introduction of an ad hoc convergence factor. In the present Appendix, some justification is given. For simplicity, only one case is considered, the calculation of $G_{-+}\left(R, \varphi ; r^{\prime}=R-x^{\prime}, \varphi^{\prime}\right)$.

From (3.5), (3.6), and the Wronskian relation,

$$
\left.G_{-+}\left(R, \varphi ; r^{\prime}, \varphi^{\prime}\right)=\frac{1}{2 \pi R} \sum_{l=0}^{\infty} \frac{I_{l}\left(m r^{\prime}\right)}{I_{l}(m R)} \exp \left[\mathrm{i}(1+l) \varphi-\mathrm{i} l \varphi^{\prime}\right)\right] .
$$

Using the uniform asymptotic expansions of the Bessel functions [29], appropriate here since the arguments $m r^{\prime}$ and $m R$ are large but the index $l$ may also be large, gives after some algebra

$$
G_{-+}\left(R, \varphi ; r^{\prime}, \varphi^{\prime}\right) \sim \frac{\mathrm{e}^{\mathrm{i} \varphi}}{2 \pi R} \sum_{l=0}^{\infty} \exp \left[-m x^{\prime}\left(1+\frac{l^{2}}{m^{2} R^{2}}\right)^{1 / 2}\right] \exp (\mathrm{i} l \theta)
$$

where $\theta=\varphi-\varphi^{\prime}$. We are interested in the $m R \rightarrow \infty$ limit of (A.2), for a fixed value of $m x^{\prime}$ and $\theta \neq 0$. For obtaining this limit, the Borel summation method is used. The sum in (A.2) can be written as

$$
S=\int_{0}^{\infty} \mathrm{d} t \exp (-t) f(t)
$$

where

$$
f(t)=\sum_{l=0}^{\infty} \exp \left[-m x^{\prime}\left(1+\frac{l^{2}}{m^{2} R^{2}}\right)^{1 / 2}\right] \exp (\mathrm{i} l \theta) \frac{t^{l}}{l !}
$$


Since the sum (A.4) is absolutely convergent, the limit and the sum can be interchanged, giving

$$
\lim _{m R \rightarrow \infty} f(t)=\exp \left(-m x^{\prime}\right) \exp \left(t \mathrm{e}^{\mathrm{i} \theta}\right)
$$

Using the limit (A.5) in (A.3) gives the large- $m R$ behaviour

$$
G_{-+}\left(R, \varphi ; R-x^{\prime}, \varphi^{\prime}\right) \sim \frac{\mathrm{e}^{-m x^{\prime}+\mathrm{i} \varphi}}{2 \pi R} \frac{1}{1-\mathrm{e}^{\mathrm{i} \theta}}
$$

in agreement with (3.15).

\section{Acknowledgements}

I have benefited of stimulating discussions with F. van Wijland. The use of the Borel summation method in the Appendix was suggested by K. Chadan. I am indebted to L. Šamaj for a critical reading of the manuscript. 


\section{References}

[1] Hansen J P, Jancovici B, and Schiff D 1972 Phys. Rev. Lett. 29991

[2] Salzberg A and Prager S 1963 J. Chem. Phys. 382587

[3] Hauge E H and Hemmer P C 1971 Phys. Norvegica 5209

[4] Kosterlitz J M and Thouless D J 1973 J. Phys. C 61181

[5] Alastuey A and Cornu F 1992 J. Stat. Phys. 66165 and references quoted there

[6] Levin Y, Xiao-jun L, and Fisher M E 1994 Phys. Rev. Lett. 732716 and references quoted there

[7] Šamaj L and Jancovici B 2002 J. Stat. Phys. 106323 and references quoted there

[8] Alastuey A and Jancovici B 1981 J. Phys.(France) 421

[9] Jancovici B 1981 Phys. Rev. Lett. 46386

[10] Cornu F and Jancovici B 1989 J. Chem. Phys. 902444

[11] Stillinger F H and Lovett R 1968 J. Chem. Phys. 491991

[12] Vieillefosse P and Hansen J P 1975 Phys. Rev. A 121106

[13] Kalinay P, Markǒs P, Šamaj L, and Travěnec I, 2000 J. Stat. Phys. 98639

[14] Hansen J P and Viot P 1985 J. Stat. Phys. 38823

[15] Jancovici B 2000 J. Stat. Phys. 100201

[16] Jancovici B, Kalinay P, and Šamaj L 2000 Physica A 279260

[17] Šamaj L and Travěnec I 2000 J. Stat. Phys. 101713

[18] Šamaj L and Jancovici B 2001 J. Stat. Phys. 103717

[19] Šamaj L 2001 J. Stat. Phys. 103737

[20] Šamaj L and Jancovici B 2002 J. Stat. Phys. 106301

[21] Jancovici B 1982 J. Stat. Phys. 2843

[22] Jancovici B 1982 J. Stat. Phys. 29263

[23] Jancovici B 1995 J. Stat. Phys. 80445

[24] Jancovici B 2002 Charge fluctuations in finite Coulomb systems (cond-mat/0201212) to be published in J. Stat. Phys.

[25] Erdélyi A 1953 Higher Transcendental Functions vol. II (McGraw-Hill: New York) 
[26] Choquard Ph, Piller B, and Rentsch R 1987 J. Stat. Phys. 46599

[27] Forrester P J and Jancovici B 1996 Int. J. Mod. Phys. A 11941

[28] Choquard Ph, Piller B, and Rentsch R 1986 J. Stat. Phys. 43197

[29] Abramowitz M and Stegun I A 1964 Handbook of Mathematical Functions (National Bureau of Standards: Washington)

[30] Choquard Ph, Piller B, Rentsch R, and Vieillefosse P 1989 J. Stat. Phys. 551185 